\title{
Políticas Interculturales de Comunicación. Una perspectiva construida desde URACCAN
}

\author{
Intercultural Communication Policies. A perspective built from URACCAN
}

\author{
Gretta Paiz Malespín
}

\section{Resumen}

En este ensayo se presentan los aspectos más destacados en el recorrido que llevó a la Universidad de las Regiones Autónomas de la Costa Caribe Nicaragüense (URACCAN) a construir su propia Política de Comunicación Intercultural y Estrategia de Comunicación Intercultural en un contexto especial de articulación, de discusión y evaluación de las metas planteadas en el Plan Estratégico Institucional 2008-2012, siendo una de estas avanzar en el proceso normativo interno comprometido.

Asimismo, poder compartir que la Política de Comunicación se fundamenta en la necesidad de mostrarle al mundo nuestra contribución al desarrollo con identidad de la región y la nación, y también, contribuir a revertir la manera en que eran tratados los temas relevantes de la Costa Caribe desde el prejuicio racial, estereotipo y la discriminación. Por tanto, es una Política que nació para desafíar y relevar la invisibilización histórica de la Costa Caribe en la representación de los medios de comunicación hegemónicos del país.

Palabras clave: Política de Comunicación Intercultural; Medios de Comunicación; Periodismo Intercultural; Prejuicios; Estereotipos.

\section{Abstract}

This essay presents the most outstanding aspects of the path that led the University of the Autonomous Regions of the Nicaraguan Caribbean Coast (URACCAN) to build its own Intercultural Communication Policy and Intercultural Communication Strategy. Both policies built in a special context of articulation, discussion and evaluation of the goals set out in the Institutional Strategic Plan 2008-2012, being one of these goals to advance the committed internal policy process.

Likewise, we can share that the Communication Policy is based on the need to show the world our contribution to development with identity of the region and the nation, and to contribute to reverse the way in which the relevant topics of the Caribbean Coast were treated. from racial prejudice, stereotype and discrimination. Therefore, a Policy was born to challenge and relieve the historical invisibility of the Caribbean Coast in the representation of the country's hegemonic media.

Key words: Intercultural Communication Policy; Communication Media, Intercultural Journalism; Prejudices, Stereotypes.

\footnotetext{
1 Máster en Salud Intercultural. Profesora Investigadora de la Universidad de las Regiones Autónomas de la Costa Caribe Nicaragüense. Correo: grettapaiz@uraccan.edu.ni ORCID: https://orcid.org/0000-0002-6036-6122
} 


\section{Introducción}

Nicaragua es un país geográficamente dividido en Pacífico y Costa Caribe. En la Costa Caribe vivimos los pueblos indígenas, afrodescendientes y mestizos costeños. Su historia de luchas y demandas llevaron al Estado nicaragüense a reconocer un día que perteneciendo a un mismo país, culturalmente éramos diferentes. Que nuestra relación comenzaba en la comunidad, con el río, el bosque, la naturaleza y el territorio. Que la organización del bienestar integral de nuestros pueblos tenía su esencia en las plantas medicinales, en nuestros médicos tradicionales y en nuestros guías espirituales.

Un territorio donde las abuelas y los abuelos son portadores de la sabiduría y el conocimiento ancestral. Un territorio que también se resistía a cualquier tipo de dominación. Y en este sentido, la educación había constituido una de las herramientas de despojo de nuestras lenguas, tradiciones y nuestra cultura. Pero fuimos un territorio que no fue entendido provocando una ruptura profunda que finalmente llevó al Estado a reconocer las demandas y derechos reivindicados desde la Ley de Autonomía, que este año cumplió 31 años. De allí que, Nicaragua es un país "multicultural" (Constitución Política, Arto.8), multilingüe y multiétnico.

En este contexto y en las entrañas de los pueblos indígenas, afrodescendientes y mestizos costeños, nace la Universidad de las Regiones Autónomas de la Costa Caribe Nicaragüense, URACCAN, en el 1995.

\section{II.- Desarrollo}

\section{De la multiculturalidad a la interculturalidad}

Todos estos años uno de los referentes importantes de interculturalidad en el país ha sido URACCAN, la Universidad comunitaria intercultural que nació con pocos recursos económicos, pero con un gran capital humano. Mujeres y hombres comprometidos con los sueños y los anhelos de gente humilde de llegar a la universidad, formarse y graduarse. En esto se expresa el modelo educativo intercultural nuestro y esta es la razón de la política de comunicación intercultural de URACCAN, visibilizar las buenas prácticas que tienen como centro sus propios protagonistas: pueblos indígenas, afrodescendientes y mestizos.

En URACCAN nos hemos propuesto pensar, dialogar y construir imaginarios y procesos que sean interculturales; su modelo educativo, su plan estratégico participativo, sus políticas, su escuela de liderazgo, sus maestrías, sus institutos y centros de investigación, su práctica de articulación y búsqueda del consenso, promueve la interculturalidad. Para URACCAN, la comunicación intercultural por ende es su columna vertebral, el sentido de responsabilidad social, una propuesta en el diálogo horizontal con la Otredad, el desafío por salir del olvido, derribar estereotipos y prejuicios (Unesco, 2014).

De este modo, resulta imposible pensar, promover y alcanzar políticas públicas interculturales en comunicación indígena y afrodescendientes sin poner en el centro de cualquier debate las asimetrías estructurales y las relaciones de poder (Asante, 1983) que se configuran en el seno de gobiernos y medios de comunicación en su afán por silenciar, invibilizar, estigmatizar, y criminalizar a los pueblos (Otero 2013, citado en Sulé, 2013).

\section{Globalización y medios de comunicación}

Estamos viviendo una era donde resulta imposible no hablar de la globalización, un proceso económico, tecnológico, de interacción, de circulación de la información, de interdependencia cultural (UNESCO, 2014), pero crucialmente también, una globalización de rupturas y de imposiciones (Ianni, 1996; 2003). En definitiva, es una globalización que evoca dominación (Gómez, 2014), y exclusión (Arriaga, Cubides y Bortolotto, 2016). 
Pero también, es una globalización que presenta la oportunidad de analizar el comportamiento de los medios de comunicación y el desafío que a estos se les presenta por comunicar, superando los prejuicios y los conflictos (UNESCO, 2014). No olvidemos que los medios configuran la opinión pública (Perucho, 2016; Alsina y Braco, 2009). En todo caso, es una globalización que evoca a repensar la comunicación desde encuadres incluyentes, promoviendo la cooperación y el respeto a la diversidad.

\section{Periodismo, prejuicios y estereotipos}

Desde URACCAN, entendemos la importancia del periodismo nacional y regional, desde la lógica de un ejercicio que contribuye a edificar sociedades más justas e igualitarias. Es una práctica que nos sugiere observarlo con respeto, pero también desde una posición crítica al momento de posicionarnos frente a temas que lesionan la dignidad de los pueblos. Nuestro planteamiento ha sido de rechazo a la lógica con la que la Costa Caribe de Nicaragua ha sido representada recurrentemente desde temas que denigran y destacan lo peor de una sociedad; narcotráfico, prostitución, delincuencia, inseguridad, escándalos políticos, inundaciones, huracanes, etcétera, etcétera. Solamente en esas condiciones éramos noticias.

En este recorrido obligado por establecer las razones que nos llevaron a construir una política de comunicación que se fundamenta en la necesidad de revertir la manera en que son tratados los temas relacionados a la Costa Caribe en los medios nacionales, recuerdo, entre otros muchos artículos y caricaturas que merecieron nuestro rechazo total, el titulado: "Reconocen Medicina de Sukias y Chamanes", en alusión a la aprobación por parte de la Asamblea Nacional, de la Ley de Medicina Tradicional y Ancestral, constitutivo del derecho, el respeto, la protección y la promoción de las prácticas y expresiones de la medicina tradicional y ancestral de pueblos indígenas y afrodescendientes en el país (Arto1: Ley 759).

De este modo, el reporte del periodista denotaba desconocimiento de lo que había intentado escribir, su información era sesgada, lo que hacía de sus argumentos imprecisos, adjetivizadores y generalizadores, algo en que no se podía confiar. La nota descontextualizaba la propia Ley a la que hacía alusión y tampoco pudo contribuir a comprender la Ley aprobada desde la claridad de los argumentos que al carecerlos, sirvió para exacerbar los prejuicios y los estereotipos del racista (Zinder, 1981) en Van Dijk (1988).

Un segundo episodio que quiero compartir, se produce en El Nuevo Diario, otro de los medios hegemónicos del país para la época, y vendría a develar el tema de los prejuicios raciales. El 24 de septiembre el 2007, este periódico desplegaba un gran titular que decía: "Guerra a los Cenis", y por ende, reproducía íntegramente la opinión del contralor de la república, Sr. Lino Hernández, planteando su desavenencia por el daño patrimonial causado por los Cenis al Estado, sosteniendo lo siguiente: “(...). porque los bancos actuaron como en una merienda de negros (...)", (Hernández 2007, citado en Ibarra, 2007).

\section{El peligro de la única historia}

Con muy poca suerte, el Sr. Contralor había invocado una frase racista que mereció el rechazo de la opinión pública. Pero, ¿qué tenían que ver los CENIS con los negros? Por supuesto que no existía ninguna relación entre lo que fue un acto calificado como el más grande daño patrimonial causado al Estado de Nicaragua. Paradójicamente, tal declaración se transformó en una reafirmación de la cultura racista, esta vez, desde la esfera de funcionarios del Estado y reproducidas en la complicidad de los medios de comunicación.

De hecho, los dos artículos redactados en distintas fechas y medios, ratificarían la carga prejuiciosa y estereotipada con la que suelen redactar algunos medios de comunicación, en abierta alusión a un sistema de prejuicios que la gente se crea respecto a los otros con un anclaje en las frustraciones, miedos, resentimientos y desconfianzas. Lastimosamente, en el mundo de los prejuicios solamente importan los aspectos negativos (Miguens, 1970) que tienen como base la ignorancia que nace en la ausencia de conocimientos y en la presencia de ideas falsas (Rose, 1970) 
Justamente y desde la postura asumida por Chimamanda Adichie, escritora Nigeriana, el peligro de una sola historia devela la configuración de estereotipos que además de falsos resultan incompletos (Adichie, 2009). La naturaleza de los estereotipos tienen expresión en cómo un grupo juzga a otros grupos (Gamarnik, 2009), en alusión a un sesgo con el que se percibe la realidad más allá de lo que la mayoría ve (Del Valle, et al, 2012). Indeclinablemente los estereotipos de los medios han profundizado las desconfianzas históricas y no han contribuido a asumir la diversidad cultural de una nación (Paiz, 2016).

\section{La formación de comunicadores}

Con estas consideraciones URACCAN se propuso formar a comunicadores interculturales vinculados a los procesos de empoderamiento y trabajo con pueblos indígenas, afrodescendientes y mestizos, y vinculados al concepto de Interculturalidad como expresión de relaciones de respeto a la diversidad, el diálogo, la escucha mutua, la concertación y la sinergia. Una comunicación que hablara de lo históricamente invisibilizado. Esta es la apuesta hoy de la maestría en comunicación intercultural: hacer visible lo que tradicionalmente nunca lo fue, desestructurar los estereotipos que denigran y descalifican a los pueblos.

De esta manera, en el 2012, nació el Proyecto de Vinculación en Comunicación Intercultural RUIICAYHIOA. Es una iniciativa educativa latinoamericana liderada por la Red de Universidades Indígenas Interculturales y Comunitarias de Abya Yala (RUIICAY), integradas por la Universidad Autónoma Indígena Intercultural (UAIIN), del Cauca Colombia, Amawtay Wasi, de Ecuador y la Universidad de las Regiones Autónomas de la Costa Caribe Nicaragüense (URACCAN) y acompañada decididamente por el colegio universitario de Oslo Noruega y NORAD.

En octubre del 2016, en el Recinto URACCAN Bluefields, se graduó el primer grupo de maestrantes procedentes de los tres países de Abya Yala (América Latina); Colombia, Ecuador y Nicaragua. Dos años después, en octubre del 2018, el segundo grupo de maestrantes se graduó en un evento internacional que tuvo como sede la Universidad Autónoma Indígena Intercultural (UAIIN) en el Cauca de Colombia.

\section{El ejercicio del periodismo intercultural}

En este contexto de formación, en el año 2013, el periodista intercultural Gilberto Artola, egresado de la maestría en comunicación intercultural y medios de comunicación en URACCAN, hoy, corresponsal de El Nuevo Diario, ese periódico del que les hablaba y que circula en la Costa Caribe desde hace más de 35 años, comenzó a denunciar desde sus artículos, la compleja problemática que se estaba viviendo en la Reserva de Biósfera de Bosawás, declarada por UNESCO Patrimonio de la Humanidad. Bosawás se encuentra anclada en territorio indígena (Musawás, en Las Minas, Región Autónoma del Caribe Norte).

Los artículos de Artola, comenzaron a insistir en la destrucción de Bosawás como referente patrimonial y pulmón de oxígeno de los pueblos indígenas que viven en sus entrañas, y de toda la humanidad. Bosawás no sólo es la segunda reserva biósfera más grande de Centroamérica y la tercera más grande a nivel de Latinoamérica, en esta parte del territorio se encuentran los sitios sagrados y las plantas medicinales con la que los pueblos indígenas aseguran el equilibrio de la salud, la espiritualidad y el bienestar integral de la gente.

La serie de artículos fue presentando a la audiencia, a las instituciones, a los movimientos ambientalistas, y demás medios de comunicación (web, redes sociales, blogeros, entre otros) información que aseguró construir y reconstruir un contexto sociocultural, de apego a la tierra y respeto a la naturaleza que tienen los pueblos, lo que significó que la gente que poco conocía sobre Bosawás, fuera construyendo también, con la información que desde los artículos de Gilberto, el Diario les daba.

El antes de Bosawás se interpreta como una etapa de invisibilización en que los medios de comunicación no conseguían integrar con igual significación a la de otros temas, procesos construidos desde la Costa Caribe de Nicaragua, su diversidad cultural, su contribución al desarrollo con identidad de un país, su sistema educativo autonómico, el modelo de salud intercultural, los curriculum construidos a la luz de las necesidades y el consenso de las comunidades, evidenciándose las tendencias de las agendas mediáticas de priorizar temas mayoritariamente políticos. 


\section{La Política de Comunicación Intercultural}

A partir de los elementos compartidos, el ejercicio de la interculturalidad promovida y comprometida por URACCAN hace posible que hoy pongamos en perspectiva la Política de Comunicación Intercultural, como la única manera de relevar la invisibilización histórica de los procesos construidos a favor una región y una nación.

De este modo, es una Política de Comunicación Intercultural que también representa nuestra contribución a la Autonomía Regional de la Costa Caribe de Nicaragua, define mecanismos ágiles de comunicación y divulgación para que lo que hacemos como institución ligada en su esencia al quehacer de los pueblos indígenas, afrodescendientes y mestizos de la Costa Caribe de Nicaragua y Abya Yala, genere en nuevas y mejores alternativas de vida (URACCAN, 2009).

Paralelamente a la Política de Comunicación Intercultural y desde el consenso de la comunidad universitaria en un proceso que involucró a amplios sectores de la sociedad costeña, URACCAN también diseñó la Estrategia de Comunicación Intercultural como una herramienta comunicacional indispensable para visibilizar los procesos construidos a nivel interno dirigidos al fortalecimiento de nuestra universidad y hacia fuera, en nuestro propio proceso de acompañamiento a las comunidades.

\section{Conclusión}

Decir, que cualquier Política de Comunicación requiere para su implementación de voluntades políticas, de talentos humanos preparados desde los propios pueblos y, sin duda alguna, de recursos financieros.

Finalmente, que la política de comunicación intercultural que hoy nuestra Universidad URACCAN pone a la disposición de los pueblos, representa el rechazo a la subalternidad como expresión de subordinación que hoy permea y configura los medios de comunicación donde el subalterno y la subalterna (los pueblos indígenas y afrodescendientes, mujeres y hombres) permanecen relegados y ausentes en el sistema representacional mediático.

\section{Lista de referencias}

Asante, M. (1983). The ideological significance of afrocentricy in intercultural communication. Journal of Black Studies, 14 (1), 3-19

Asamblea Nacional (1987). Ley 28. Estatuto de la Autonomía de las Regiones de la Costa Atlántica de Nicaragua. Recuperado en: http://legislacion.asamblea.gob.ni/Normaweb. nsf/\%28\$All\%29/9F88A9114C4CA12Fo62570A100578099?OpenDocument.

Asamblea Nacional (2003). Decreto 3584. Reglamento a la Ley No. 28. Estatuto de Autonomía de las Regiones de la Costa Atlántica de Nicaragua. Recuperado en: http://revistas.bicu.edu.ni/index.php/wani/ article/download/474/470.

Asamblea Nacional (2011). Ley 754. Ley de Medicina Tradicional Ancestral. Recuperado en: http://www. calpi-nicaragua.org/ley-de-medicina-tradicional-ancestral/.

Asamblea Nacional (2014). Constitución Política de Nicaragua. Recuperado en: http://www.asamblea.gob. $\mathrm{ni} / \#$.

Adichie, C. (2009). El peligro de la historia única. Recuperado en: https://www.ted.com/talks/ chimamanda_adichie_the_danger_of_a_single_story?language $=$ es 
Campbell, M. (S.F). Afrodescendientes en las Américas. De la esclavitud a la visibilización: 500 años de exclusión y marginalización.

Carmona, DP. (S.F). Medios de comunicación, identidad e ideología. Flacso, Ecuador, pp.1-10.

Del Valle, Carvajal, Orozco, Jurado y Madrid. (2012). Sobre el peligro de una sola historia: Análisis de las premisas del interaccionismo simbólico. Poiésis, Revista Electrónica de Psicología Social. (24), 1-4.

González, G.(1999). Los estereotipos como factor de socialización en el género. Comunicar 12, 79-88.

Grimson, A. (2001). Introducción, pp. 13-19. En Grimson, A. (2001). Interculturalidad y Comunicación. Grupo Editorial Norma.

Gamarnik, C. (2009). Estereotipos Sociales y Medios de Comunicación: Un Círculo Vicioso.

Hooker, A. (2010). 13 años de URACCAN en Nueva Guinea. Posible al esfuerzo y compromiso de su Pueblo. URACCAN AL DÍA, (10), agosto-diciembre.

Hooker, A. (2010). URACCAN entrega a la sociedad de Nueva Guinea nuevos profesionales. Constructores de sueños y esperanzas. URACCAN AL DÍA, (10), agosto-diciembre.

Ibarra, E. (24 de septiembre del 2007). Guerra de los Cenis. El Nuevo Diario de Nicaragua.

Migueen, J. (1970). Prólogo, p. 9-18. En: Rose, A. (1970). El origen de los prejuicios. Editorial Hvmanitas, Buenos Aires, Argentina.

Sulé, J. (2013). Vicente Otero, periodismo Indígena. Recuperado en: https://javiersule.wordpress.

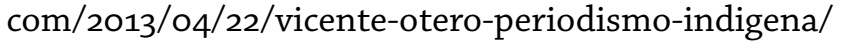

Rose, A. (1970). La ignorancia acerca de otros grupos humanos, causa de prejuicios, pp. 29-34. En: Rose, A. (1970). El origen de los prejuicios. Editorial Hvmanitas, Buenos Aires, Argentina.

Tijerino, J.C. (17 de julio, 2011). Reconocen medicina de sukias y chamanes. Diario La Prensa de Nicaragua.

Paiz, G. (2016). Evaluando la calidad en la maestría internacional en comunicación intercultural con enfoque de género, de la Red de Universidades Indígenas Interculturales y Comunitarias de Abya Yala (RUIICAY). Revista Caribe, (17), 7-19.

Paiz, G. (2016). Tejiendo el consenso desde la combinación de las agendas. La agenda melding. Ciencia e Interculturalidad, Vol. 19, 9 (2), 104-121.

URACCAN (2009). Política de Comunicación Intercultural. Universidad de las Regiones Autónomas de la Costa Caribe Nicaragüense (URACCAN).

URACCAN (2010). Política Intercultural de Género de URACCAN.

UNESCO (2014). La función de los medios de comunicación en el diálogo intercultural.

URACCAN (2014). Plan Estratégico Participativo de la URACCAN (PEI) 2015-2019. "Construyendo el camino de la educación intercultural comunitaria con calidad”. Managua, Nicaragua, URACCAN.

Van Dijk, T. (2008). Semántica del discurso e ideología. Discurso \& Sociedad, 2(1), 201-261. 